\title{
FlocARAZI: an in-situ, image-based profiling instrument for sizing solid and flocculated suspended sediment
}

\author{
Ryan Osborn ${ }^{1}$, Brandon Dillon ${ }^{1}$, Duc Tran ${ }^{2}$, Ehsan Abolfazli ${ }^{1}$, Kieran B.J. \\ Dunne $^{3}$, Jeffrey A. Nittrouer ${ }^{4}$, and Kyle Strom ${ }^{1}$ \\ ${ }^{1}$ Civil and Environmental Engineering, Virginia Tech, Blacksburg VA USA \\ ${ }^{2}$ IFREMER, DYNECO/DHYSED, ZI pointe du Diable, CS10070 Plouzan, France \\ ${ }^{3}$ Caltech, Division of Geological and Planetary Sciences,Pasadena, CA, USA \\ ${ }^{4}$ Texas Tech University, Department of Geosciences, Lubbock, TX, USA
}

Key Points:

- A low-cost underwater camera system is developed for in-situ profile imaging of flocculated mud and other suspended particles.

- The camera system can provide accurate measurement of suspended particle size distributions in the size range of 5 to 600 microns.

- Methods are introduced to separate flocculated mud from sand in the sizing and to estimate the mass concentration of each fraction.

Corresponding author: Kyle Strom, strom@vt.edu

This article has been accepted for publication and ${ }^{-1}$ undergone full peer review but has not been through the copyediting, typesetting, pagination and proofreading process, which may lead to differences between this version and the Version of Record. Please cite this article as doi: 10.1029/2021JF006210.

This article is protected by copyright. All rights reserved. 


\begin{abstract}
An inexpensive and compact underwater digital camera imaging system was developed to collect in situ high resolution images of flocculated suspended sediment at depths of up to 60 meters. The camera has a field of view of $3.7 \times 2.8 \mathrm{~mm}$ and can resolve particles down to $5 \mu \mathrm{m}$. Depending on the degree of flocculation, the system is capable of accurately sizing particles to concentrations up to $500 \mathrm{mg} / \mathrm{L}$. The system is fast enough to allow for profiling whereby size distributions of suspended particles and flocs can be provided at multiple verticals within the water column over a relatively short amount of time (approximately 15 minutes for a profile of 15 meters). Using output from image processing routines, methods are introduced to estimate the mass suspended sediment concentration (SSC) from the images and to separate identified particles into sand and mud floc populations. The combination of these two methods allows for the size and concentration estimates of each fraction independently. The camera and image analysis methods are used in both the laboratory and the Mississippi River for development and testing. Output from both settings are presented in the paper.
\end{abstract}

\title{
Plain Language Summary
}

Rivers carry large quantities of muddy sediment that finds its way into river beds, floodplains, lakes, reservoirs, and coastal water bodies. Scientists and engineers rely on measurements of sediment size to understand how sediment moves in a river and where it ultimately deposits. Yet, measurement of muddy sediments (sediment with particle diameters $<63$ microns) has proven difficult to accomplish. Reasons for this include the small size of the sediment and the ability of small particles to stick together to form aggregates that change size depending on the conditions in the flow. For this reason, accurate measurement of the hydrodynamic size of mud cannot be achieved by sizing samples in the lab. In this paper, we present an imaging system we developed to measure muddy sediment aggregates, or flocs, within natural flows. The system is relatively inexpensive, reproducible, and is capable of providing higher spatial and temporal resolution of the suspended particle sizes than any other imaging system previously developed. In the paper we describe the system, test and validate it in the laboratory and field, and show how the data can be processed to provide unique information about the type and amount of sediment available in the water column.

\section{Introduction}

\subsection{Overview}

Mud (mixtures of inorganic and organic fine sand, silt, and clay) can constitute a significant fraction of sediment transported by rivers to deltaic zones. For this reason, the fate of mud has many implications for the health of deltaic systems, maintenance of navigation channels, and future planning of coastal restoration projects. Yet both the measurement and modeling of this sediment fraction has proven difficult to accomplish. One reason for this difficulty is the difference in light and sound scattering properties between solid particles (such as mineral grains of silt and sand) and the irregularly shaped porous aggregates of fine sediment found in the water column of muddy systems known as flocs (Fig. 1). Flocs can form due to the presence of fine clay and silt and particle attached biofilms (Eisma, 1986; Droppo \& Ongley, 1994). In addition to the problems associated with the difference in density and shape between these two forms of suspended matter that exists in these systems (and the associated differences in light and sound scattering properties that result), the porous mud flocs also have the ability to change their size and shape as they go through different hydrodynamic and biological and ionic conditions in the water column (e.g., Mietta et al., 2009). 

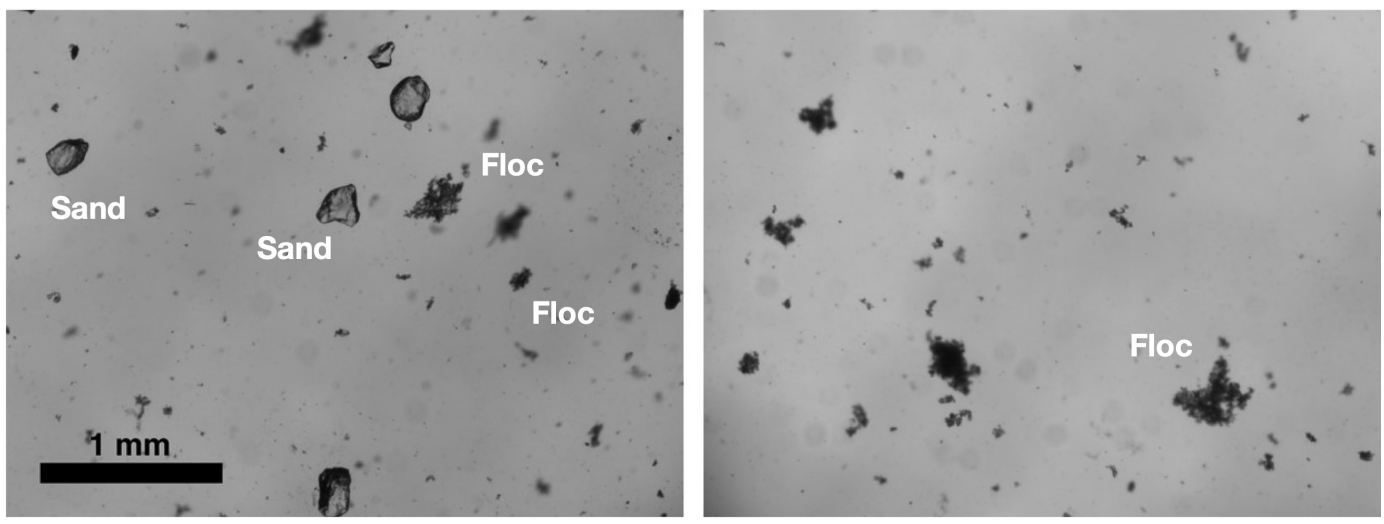

Figure 1. Example images collected with the FlocARAZI (the instrument detailed in this paper) while deployed in the Mississippi River. Both sand and flocs are present in the images on the left with some of the sand grains and flocs labeled. The image on the right does not contain any solid sand particles.

Measurement and modeling of the flux of muddy fine sediment requires information regarding the mass or volume concentration of the sediment and its settling velocity. For solid unaggregated silt and sand, the settling velocity of the material is related to the grain size in a straightforward way through a settling velocity equation (e.g., Ferguson \& Church, 2004, or Stokes settling velocity). Furthermore, the size of such material does not change with time or local flow conditions. Therefore, physical samples of suspended sand can be taken to the laboratory to measure both the concentration and size of the sediment, and thereby, settling velocity. However, for flocculated mud, the settling velocity is related to both the floc size, effective density, and the floc shape and porosity - all of which can change rapidly in response to local changes in the water column. For these reasons, it is not possible to take a physical water sample of mud from the field and then use classic methods of sizing or settling velocity estimation (such as a laser diffraction instrument or settling column) in the lab or even onboard a vessel to obtain the information needed to characterize the settling velocity of the flocculated mud fraction without the possibility of the floc properties measured being different than those that existed within the water column. Instead in situ measurements must be made to obtain the most accurate characterization of the mud. Characterization of the mud can be accomplished through measurement of the floc size (with density estimated in some way) or through measurement of the floc size and settling velocity.

A range of methods have been employed to characterize flocs in situ. Such methods include in-situ settling columns combined with imaging systems to measure floc size and settling velocity, in-situ imaging devices for measuring floc size, and laser-based methods for measuring particle size and volume concentration such as the Sequoia Scientific Laser In-situ Scattering and Transmissometry (LISST) 100x and 200x (Agrawal \& Pottsmith, 2000). Of these, the most commonly used instrument is the commercially available LISST 100x and 200x. While the LISST family of instruments are extremely useful, differences in light scattering properties between solid grains and porous flocs can lead to ambiguity in the ability of the LISST to resolve the true size of flocculated mud. This uncertainty arises because Mie small-angle forward scattering (the theory on which LISST measurements are based) cannot always accurately measure the true absolute size of large, irregularly-shaped flocs of variable density (Mikkelsen et al., 2005; Smith \& Friedrichs, 2011). Furthermore, LISSTs may provide ambiguous data in salinity driven pycnoclines where the Schlieren effect can influence measured particle sizes (Mikkelsen et al., 2008; Karageorgis et al., 2015; Chapalain et al., 2019). For these reasons, image-based instru- 
ments are needed as a complement to or in place of a LISST when the aim of the work is to characterize the flocculation state of mud (Mikkelsen et al., 2005, 2006; Davies \& Nepstad, 2017; Fall et al., 2021). However, to date, a commercial imaging system suitable for characterizing flocs at concentrations typical of riverine and estuarine environments does not exist. For this reason, specialty in situ imaging systems are typically developed by individual research groups to measure flocs (e.g., Benson \& French, 2007; Smith \& Friedrichs, 2011; MacDonald \& Mullarney, 2015).

In this paper we introduce a new low-cost, in-situ profiling floc imaging and imageprocessing system capable of providing separate size populations of suspended mud (both flocs and solid particles) and sand in the size range of 5 to $600 \mu \mathrm{m}$ (in the current configuration) at a higher resolution and frequency than has been accomplished in the past. While the system is not commercially available, the system has been built with off-theshelf and 3D printed parts in an effort to make it easier for others to build and improve upon. The next section of the paper presents a brief review of other imaging devices that have been developed for either the measurement of floc size or to obtain floc size and settling velocity. We then highlight the more specific purpose of our camera system, describe the parts package, and outline the testing of the instrument and the processing procedures used to extract the data.

\subsection{Background: floc imaging systems}

Imaging systems that have been developed to characterize flocs can broadly be grouped into those that provide only floc size and those that aim to measure floc size and settling velocity. Other differences include whether the system is designed for deployment at a single depth or for vertical profile measurements, whether or not there is realtime feedback between the camera and operator, how the image is illuminated, the speed of image acquisition and processing, and the range of sizes that can be accurately measured. Below we briefly summarize some of the systems that have been designed to provide the context for the system we are presenting in this paper. The section is not intended as an exhaustive review of all methods used for characterizing flocs, but instead focuses on those instruments most like ours.

In-situ, image-based settling column and particle sizing systems aim to minimize the disruption to flocs and provide information on their size and density, which is closely tied to the environmental conditions in which they exist. The addition of imaging sediment within the settling column provides the means for a direct measurement of floc sizes and settling velocity through particle tracking. Examples of settling column imaging systems include the IN Situ SEttling Velocity instrument (INSSEV) (Fennessy et al., 1994) and the Particle Imaging Camera System (PICS) (Smith \& Friedrichs, 2011). Images from the INSSEV system capture settling velocity directly by tracking particle motion frame-by-frame. This method requires that disturbances to the system be minimized to reduce external influence on the particles settling within the settling column. Stability with the INSSEV system is maintained by locating the system on a weighted tripod that is positioned on the bed during sampling (Manning \& Dyer, 2002). Consequently, the system is limited to measuring particle size and settling velocity at the level of the tripod. The PICS is capable of estimating settling velocity and particle size over the full range of the water column. The system can be lowered to a desired depth, where a water sample is collected within the settling column and separated from the external flow by closing both ends of the settling column. Images are then collected after the settling column is closed. Estimating floc settling velocity with this systems is achieved by employing particle tracking velocimetry (PTV) to measure the velocity of individual flocs, and particle image velocimetry (PIV) to estimate the background fluid velocity by tracking particles smaller than 2 pixels (Smith \& Friedrichs, 2015). For a series of images, the settling velocity for a floc is estimated as the vertical component of the average vector 
subtraction of the fluid velocity from the floc velocity. The resolution of the INSSEV and PICS is approximately 20 to $900 \mu \mathrm{m}$ and 30 to $1000 \mu \mathrm{m}$, respectively.

These systems were designed for the purpose of measuring floc size and settling velocity at targeted locations. However, the nature of settling velocity measurements requires longer periods of time to collect measurements at a particular field location compared to collecting particle size information alone, as can be done with a device such as a LISST-100x. Furthermore, making direct settling velocity measurements of slow settling mud is inherently difficult, even in the lab (Tran \& Strom, 2017), and adding this measurement capability to a system increases the design complexity and cost. For these reasons, other researchers have sought to build imaging devises that focus on measuring only the size of the suspended aggregates.

Notable examples of such systems designed to measure only floc size include the Digital Floc Camera (DFC) of Curran et al. (2003), Mikkelsen et al. (2004) and Hill et al. (2011), the In situ Particle Imaging Device (InSiPID) of Benson and French (2007), the Remote In-situ Particle Settling Camera (RIPScam) of Cartwright et al. (2011), the DFC of Mikkelsen et al. (2008), the FlocDrifter platform of MacDonald and Mullarney (2015), and the Pcam system of Markussen et al. (2016). All of these systems take a slightly different approach to acquiring floc images, but all aim to photograph flocs within the water column and then use image processing to extract the size of the imaged particles. Sequoia Scientific's LISST-HOLO (Graham et al., 2012) is another device that can be used to image flocs. The devices uses holography to provide $3 \mathrm{D}$ reconstructed images of irregularly shaped particles. While the LISST-HOLO does provide imaged 3D particle data, the method and instrument is more suited to very low concentration environments such as might be found in coastal or open ocean settings.

Key questions and issues to overcome when building floc imaging systems include: (1) what type of camera and lens combination to use (implications for image resolution and maximum field of view, power needs, settings control, and image storage); (2) how to provide adequate illumination for the image while either having a fast enough shutter speed or short enough exposure time to prevent streaking of the moving particles in the image; and (3) how to extract accurate size information from the images in a timely way.

All of the systems listed above have taken different approaches when addressing these key questions and issues. For example, some systems use larger digital single-lens reex (DSLR) cameras with macro lenses (e.g., Cartwright et al., 2011; MacDonald \& Mullarney, 2015; Markussen et al., 2016). An advantage of this approach is that the cameras come with built in controls and the availability of local power and data storage. These systems however also have their drawbacks in that they are larger, may have limitations on the length of cord that can be used to control them externally, and have limitations on the magnification level of available lenses. Another approach is to use smaller scientificgrade digital cameras that can be fitted with microscope lenses (e.g., Mikkelsen et al., 2004; Benson \& French, 2007). Such systems are more suitable to collecting high-quality images of small objects, are setup for external control, and have an overall smaller footprint which could make them easier to waterproof. However, drawbacks to this approach include the need to provide power, camera control, and data storage remote to the camera itself. This can be accomplished with microcontrollers, solid state hard drives, and battery packs, or through an online remote connection to power and a computer.

A combination of the camera sensor size and resolution, and the level of lens magnification, ultimately sets the size range of particles that can be imaged with any of these systems. The key trade off to weigh when selecting lens magnification is that between resolution and field of view. Higher magnification lenses provide more resolution, but limits the field of view. Furthermore, as magnification increases, freezing moving particles in the image becomes increasingly difficult due to the small field of view and increased 
light needed to adequately expose the images. A unique approach to dealing with the resolution versus field of view trade off was used by Benson and French (2007). Rather than choosing a single camera sensor and lens combination, they used two different cameras with lenses of different magnification to produce a measurable range of $4-3000 \mu \mathrm{m}$ with their InSiPID device.

The two-camera system is appealing for the increased range of particle sizes that can be measured, but it is also more complicated. For this reason, most floc imaging systems have used a single image sensor and lens combination. For earlier systems this produced a rather limited range of particle sizes that could be measured. For example, the instrumentation package of Mikkelsen et al. (2004) used a DFC with a 1024 x 1024 pixel sensor that could only measure particles down to $135 \mu \mathrm{m}$ in diameter with a image pixel resolution of $45 \mu \mathrm{m}$ per pixel. While the system was novel at the time, improvements in camera sensors now provide the opportunity for much higher resolution images. When coupled with the right optics these can provide much better resolution. For example both the RIPScam of Cartwright et al. (2011) and the Pcam of Markussen et al. (2016) have camera sensor and optics packages that result in image pixel sizes of approximately 4 $\mu \mathrm{m}$ and the ability to measure flocs with diameters ranging from $20 \mu \mathrm{m}$ to several $\mathrm{mm}$ (up to $20 \mathrm{~mm}$ in the case of the RIPScam). The FlocDrifter platform (MacDonald \& Mullarney, 2015) was capable of shifting this range to smaller sizes with image pixel sizes of $1.5 \mu \mathrm{m}$ and field of view of $7.7 \times 5.1 \mathrm{~mm}$; effectively allowing them to measure sizes between $10 \mu \mathrm{m}$ and $5 \mathrm{~mm}$.

Questions surrounding illumination of the image come in two basic forms. The first is, should bright field, dark field, or laser-sheet illumination be used? The second is, what method should be employed to adequately expose the image while keeping streaking from moving particles in the image to a minimum? With regard to the first question, most have tended to use bright-field illumination (i.e., backlit images). This method produces silhouettes of darker suspended particles within a more illuminated fluid background. The exception to this is the Pcam of Markussen et al. (2016) in which illumination was provided by a laser sheet passing through the field of view. This method produces bright particles on a dark background similar to classic particle image velocimetry. For the second, two basic approaches have been used. The first is to image a sample of the suspension that has been physically removed from the ambient flow to reduce the speed of particles relative to the imaging system (e.g., the RIPScam). The second has been to use a strobed light source either in combination with a stilling chamber when suspended samples can freely flow through the imaging region (Benson \& French, 2007; MacDonald \& Mullarney, 2015). For slow moving particles, a bright constant light source has also been used (Mikkelsen et al., 2004).

All of the imaging methods discussed above require that particles be identified and measured from the set of images collected. The volume of data these methods can produce, especially with modern cameras, means that the extraction of data needs to be done using automated image processing with minimal user input. While care must be taken to build a set of processing routines that accurately measures the particles that are there in the image (while removing poorly image particles from the output), this task can often be accomplished in a reasonable way as long as good images can be collected (Keyvani \& Strom, 2013; Smith \& Friedrichs, 2015).

\subsection{Objectives}

Imaging aggregates in situ remains the most accurate method for sizing mud flocs. Yet, no commercial instrumentation exist to accomplish this task, and each research team is left to develop their own system. Furthermore, many of the systems that have been created in the past rely on specially machined parts and/or microcontroller programing that make it more difficult and costly to reproduce. 
In this paper we introduce a new, low cost and compact suspended sediment imaging system called the FlocARAZI (Floc AReA and siZing Instrument). The FlocARAZI is designed to image flocculated sediment that is allowed to pass freely through a variablewidth flow-through cell, thereby minimizing interaction of sediment with the device and allowing for continuous profiling of suspended sediment over the water column in riverine and estuarine environments. Power and signal to the camera, and a continuously illuminated light source, are controlled at the surface through two ethernet cables, allowing for real-time monitoring of the camera feed during deployment. Use of off-the-shelf and 3D printed parts helps to keep the overall cost of the system low and to make the system more easily reproduced by others.

In this paper we also present two methods that use the output of a previously developed image processing routine to provide unique information about the suspension. First, a method is introduced to estimate the mass suspended sediment concentration (SSC) of flocculated sediment from the collected image data. The ability to estimate SSC from FlocARAZI image data provides the means to collect higher resolution SSC information over the water column than could be obtained with physical water samples alone. The second processing method utilizes floc and sand size and texture properties to train a support vector machine (SVM) learning algorithm to classify suspended sediment particles as either sand or flocculated mud. The ability to distinguish between flocs and sand is a key benefit of in-situ imaging devices, especially when properties of flocs, rather than the full range of particles in suspension are of interest. Once trained, the learning algorithm provides an accurate way to quickly identify sand within the particle data output from the image processing routine.

Details pertaining to the imaging system build are presented in the next section (section 2). Following these details we present data used to test the overall ability of the instrument to measure particle sizes accurately (imaging and image processing) and discuss a sample deployment on the Mississippi River. In section 4 we provide details pertaining to SSC estimation from the images and show the functionality and calibration of the system for a detailed lab experiment and a field application. Section 5 focuses on the classification of identified particles as pieces of sand or as mud flocs. Data for this classification is obtained from both the laboratory and field.

\section{FlocARAZI}

The FlocARAZI (Fig. 2) consists of a camera mounted to a stepper-motor-driven linear slide rail stage, and an LED for illuminating the camera field of view all contained within a waterproof housing. The camera, stepper motor, and LED are powered and controlled from the surface by two 60-meter-long weatherproof Cat6 ethernet cables. A direct connection to the surface allows for a real-time camera feed and in-situ adjustment of the camera focus and LED brightness. The camera is a monochrome $4000 \mathrm{x} 3000$ pixel FLIR Blackfly S GigE with a CMOS Sony IMX226 sensor that has a pixel size of 1.85 $\mu \mathrm{m}$, and is capable of collecting images at a rate of up to 10 frames per second. The camera lens assembly consists of a 5X Mitutoyo plan apochromat, infinity corrected, long working distance objective mounted to a ring-actuated aperture stopped down to f/21.4. The maximum $\mathrm{f}$-number of the system is $\mathrm{f} / 6.3$. The objective is positioned at the end of a $78 \mathrm{~mm}$ SM1 threaded tube containing an achromatic doublet lens with a focal length of $75 \mathrm{~mm}$. The combination of the 5X Mitutoyo objective with the achromatic doublet lens produces an effective magnification of $2 \mathrm{X}$, resulting in an image pixel size of 0.925 $\mu \mathrm{m}$ and a nominal field of view of $3.7 \times 2.8 \mathrm{~mm}$. A stepper-motor-driven $150 \mathrm{~mm}$ linear slide rail allows for focusing of the camera during deployment. The LED is a $6 \mathrm{~V}$ CREE Mt-G2 Q0 on a Noctigon Mt-G20 MCPCP rated for a maximum nominal light output of 1990 lumens at 18.5 watts. 

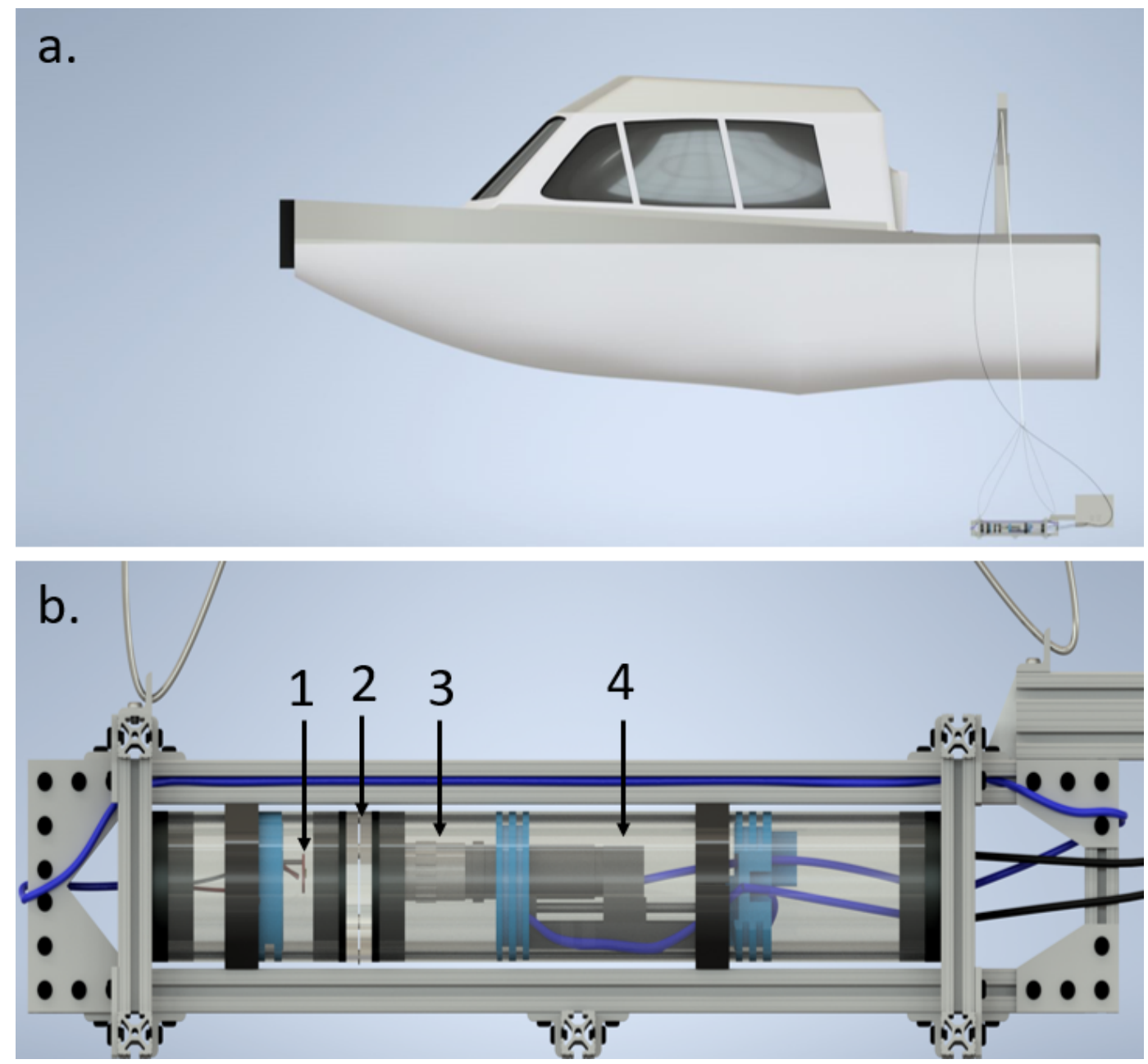

Figure 2. The FlocARAZI both (a) in its deployed state and (b) a close up side view identifying the (1) LED, (2) $1.17 \mathrm{~mm}$ flow-through cell, (3) Mitutoyo objective and lens assembly, (4) camera mounted to a slide rail stage. The black wires entering the camera tube control the linear slide rail stepper motor and send a live video feed to the surface. 
The camera assembly and LED are housed in separate BlueRobotics 3-inch diameter watertight acrylic tubes that are rated to $150 \mathrm{~m}$ depth. A flow-through cell consisting of two acrylic end caps with a gap through which water and sediment are free to flow, separates the camera and LED tubes (Fig. 2). The acrylic end caps are secured together in concentric alignment with six tapered stainless-steel screws positioned within six equally spaced through holes along the perimeter of the acrylic end caps. The gap width can be adjusted for different applications by placing spacers of the desired thickness between the acrylic end caps before tightening the screws; the default gap size is $1.17 \mathrm{~mm}$, but this can easily be adjusted to larger or smaller sizes. The camera is set to focus on the center of the flow-through cell gap by means of adjusting the linear slide rail stage position through controlling the stepper motor. One-inch aluminum T-Slotted framing rails are used to support the acrylic tubes and provide mounting points for a hoist connection and additional equipment. To maintain a consistent orientation into the flow, a 36.5 $\mathrm{x} 31 \mathrm{~cm}, 1.5 \mathrm{~mm}$ thick aluminum plate is mounted to the framing to act as a rudder.

While the FlocARAZI is deployed, the camera is powered and transmits a live video feed through one of the two 60-meter-long Cat6 ethernet cables, with power supplied by a power over ethernet injector. The video feed is displayed and recorded through the FLIR SpinView GUI. The second ethernet cable supplies a signal to the stepper motor, for controlling the linear slide rail platform position, and power to the LED. Power is supplied to the LED by a DC power supply through four of the 8 available ethernet wires, which allows for adjustment of the brightness by changing the current supplied to the LED. Power to the stepper motor is supplied by an onboard $7.4 \mathrm{~V}, 1500 \mathrm{mAh}$ battery. The remaining 4 wires in the second ethernet cable send direction and speed controls to the stepper motor for focusing the camera.

\section{Validation and Test Deployment}

\subsection{Sizing: Image Processing and Validation}

The ability of the camera system and processing routine to resolve and accurately size particles in suspension was tested by conducting controlled laboratory experiments in which PIV seeding particles were imaged. The images were then processed to obtain a size distribution that was compared to a particle size distribution obtained by sizing the seeding particles with a HORIBA LA-300 laser scattering particle-size distribution analyzer. The image processing routine was then updated to best match the camera-produced size distribution with that from the HORIBA LA-300. Due to the relatively large size of the FlocARAZI compared to available testing tanks, the modified setup shown in figure 3 had to be used. The setup consists of a 13-liter acrylic mixing tank, and the same LED light source and camera assembly described in the previous section. The spacing of the flow-through cell was maintained by fixing the same $1.17 \mathrm{~mm}$ spacers between the LED light source and the wall of the mixing tank, though the actual width was measured to be $1.55 \mathrm{~mm}$ due to added thickness from adhesive material.

Images for these experiments and others are processed following the general method of Keyvani and Strom (2013), which employs an automated script that identifies all particles within an image, via an ImageJ (Schneider et al., 2012) macro, and removes outof-focus particles within MATLAB R2013B. The ImageJ macro provides individual particle information such as area, perimeter, fit ellipse dimensions, shape descriptors, bounding box dimensions, and particle location within the image, for each image. The MATLAB script then uses the previously obtained particle location to obtain the pixel intensity matrix of the bounding box for each particle within the image set, and determines the clarity and contrast of each particle. The clarity of a particle is a measure of the steepness of the pixel grey scale values near the edge of the particle. The contrast is a measure of the difference in pixel intensity for the pixels that make up the particle. Particles with a clarity value greater than 0.7 were assumed to be in focus and their infor- 


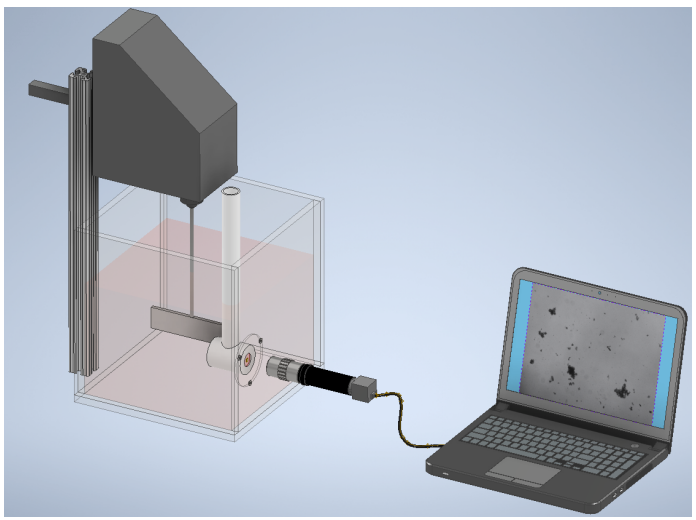

Figure 3. Mixing tank setup used for validating the FlocARAZI camera. The same $1.17 \mathrm{~mm}$ flow-through cell spacers were used in an attempt to mimic the flow-through cell present on the FlocARAZI.

mation was retained. For those particles deemed in focus, the equivalent circular diameter of each floc or solid particle (in pixels), $d_{f}$, is calculated within each image from the measured floc area, $A$, as:

$$
d_{f}=\sqrt{\frac{4 A}{\pi}}
$$

A few minor changes were made to the process of Keyvani and Strom (2013) to account for increased image resolution of the FlocARAZI camera compared to the camera used in their study. In the background subtraction step, the rolling ball radius was increased from 50 to 60 pixels and the sliding paraboloid function was included. Additionally, the triangle thresholding function was used instead of the Yen method (Zack et al., 1977). It was determined through trial and error that this combination of rolling ball radius and the sliding paraboloid function with the triangle thresholding was the most robust method for sizing particles in the range of $5600 \mu \mathrm{m}$. The last change to the original processing routine was to include a binary erode step after thresholding. The erode function removes one layer of pixels from the edges of objects within the thresholded image. This step was included so that the outline of the particles identified by ImageJ better matched the expected outline based on the original image.

We determined the lateral resolution of the microscope, as configured, to be 0.925 $\mu \mathrm{m} /$ pixel. This calibration was found by using a stage micrometer slide with ruled divisions of $0.1 \mathrm{~mm}$. On average, 1081.1 pixels were required to image 10 divisions $(1 \mathrm{~mm})$ on the reticule.

Validation of the pixel to micrometer conversion and the image processing routine was performed by comparing the camera produced size distribution of 10 and $20 \mu \mathrm{m}$ PIV seeding particles to the size distribution obtained by a HORIBA LA-300 (Fig. 4). The HORIBA LA-300 is a laser scattering particle-size distribution analyzer that is capable of measuring particles in the range of 0.1 to $600 \mu \mathrm{m}$. The particles used for the tests were Dantec Dynamics $10 \mu \mathrm{m}$ Silver Coated Hollow Glass Spheres (S-HGS) and $20 \mu \mathrm{m}$ Polyamide Seeding Particles (PSP). According to Dantec Dynamics, the S-HGS have a mean particle size of $10 \mu \mathrm{m}$ and a size distribution of 2-20 $\mu \mathrm{m}$ and the PSP have a mean particle size of $20 \mu \mathrm{m}$ and a distribution of 5-35 $\mu \mathrm{m}$. Preparation of each sample for sizing with the FlocARAZI camera consisted of sonicating $30 \mathrm{mg}$ of the seeding particles within a $30 \mathrm{~mL}$ vial of tap water. The sample was then added to the 13-liter mixing tank. The paddle speed in the mixing tank was set to a mixing rate of $50 \mathrm{rpms}$, corresponding to a turbulent shear rate of $90 \mathrm{~Hz}$, which is within the mixing rate range for previous floc 
studies (Kumar et al., 2010; Mietta et al., 2009; Zhu et al., 2015). The camera collected one image every second for 20 minutes. A size distribution was created (Fig. 4) for the 20 minutes of image size data following the aforementioned image processing routine. This process was carried out for both the S-HGS and PSP suspensions. Preparation of the sample for sizing with the HORIBA LA-300 followed a similar procedure, however, the sample was first diluted to a concentration suitable to achieve the light transmission requirements of the system. The seeding particles in the suspension were measured with the HORIBA LA-300 three separate times and the resulting distributions were averaged to obtain the particle size distributions presented in figure 4. The size distribution produced by the FlocARAZI camera and the HORIBA LA-300 are quite similar, with only a slight difference in the mean particle size $(<1 \mu \mathrm{m})$ and standard deviation $(<1 \mu \mathrm{m})$ (Table 1).
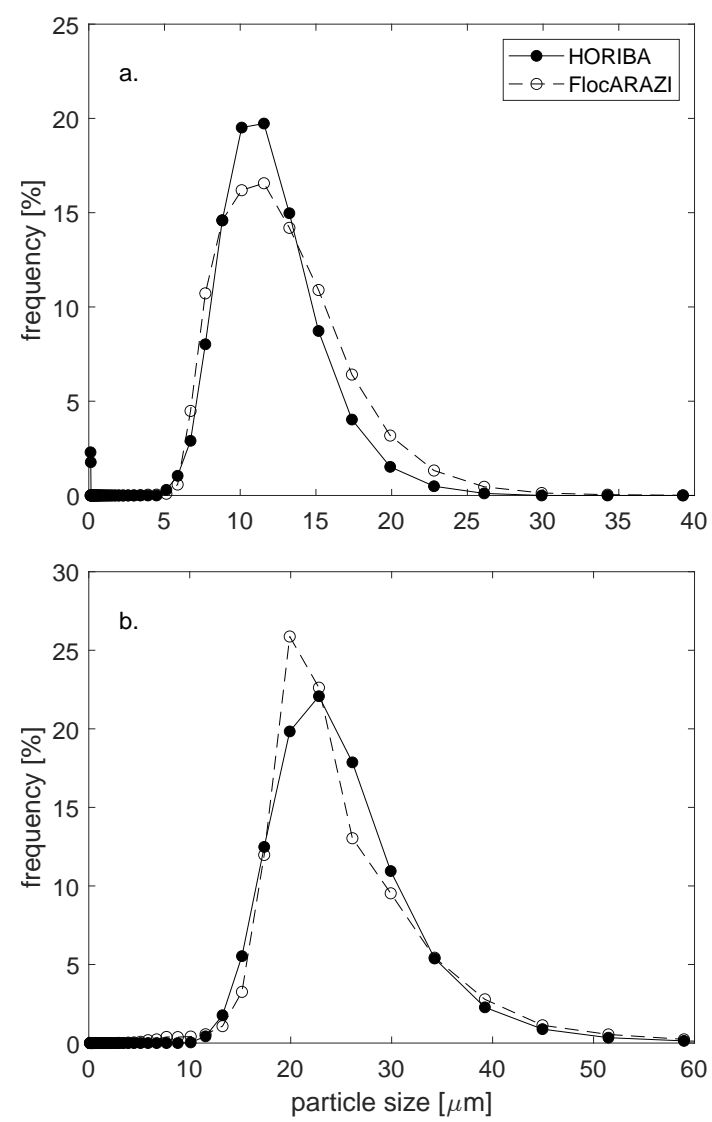

Figure 4. Size distribution comparison between (a) $10 \mu \mathrm{m}$ and (b) $20 \mu \mathrm{m}$ seeding particles sized with a HORIBA LA-300 and FlocARAZI.

Table 1. Particle sizing with HORIBA LA-300 and FlocARAZI camera

\begin{tabular}{cccc}
\hline $\begin{array}{c}\text { Particle Size } \\
{[\mu \mathrm{m}]}\end{array}$ & Sizer & $\begin{array}{c}\text { Mean } \\
{[\mu \mathrm{m}]}\end{array}$ & $\begin{array}{c}\text { Std. } \\
{[\mu \mathrm{m}]}\end{array}$ \\
\hline \multirow{2}{*}{10} & HORIBA & 10.9 & 3.7 \\
& FlocARAZI & 11.8 & 3.8 \\
20 & HORIBA & 23.6 & 6.3 \\
& FlocARAZI & 23.5 & 7.2 \\
\hline
\end{tabular}


The comparison outlined above shows that, with proper calibration of the conversion from camera pixel length to physical length and the image processing routine, the FlocARAZI can produced particle size distributions that compare favorably to those of the HORIBA LA-300. Additional testing with the camera in the same lab setting has produced favorable results for flocs in the size range of $50-85 \mu \mathrm{m}$ when compared to older lab cameras that have previously been used and calibrated (Keyvani \& Strom, 2013; Tran \& Strom, 2017). The upper limit of the particle size range that the camera system can accurately capture is dependent on the width of the flow-through cell gap and the field of view of the camera. Therefore, the width of the flow-through cell gap should be adjusted depending on the floc size range and overall suspended sediment concentration expected to be imaged. With the current flow-through cell gap and good lighting, the FlocARAZI camera system can accurately size particles in the range of $5-600 \mu \mathrm{m} ; 600$ $\mu \mathrm{m}$ is approximately one-half of the gap size (Tran et al., 2018).

\subsection{Field Deployment Example}

Validation of the FlocARAZI's operation in the field has been tested through deployments of the system within the lowermost sections of the Mississippi River. The FlocARAZI has been successfully deployed to depths of up to 36.5 meters. During these deployments, images collected with the FlocARAZI (Fig. 1) have provided floc size information within the main channel of the Mississippi River, its distributaries, and the plume within the Gulf of Mexico at the exit of South and Southwest Pass. Images were collected at a rate of 2 images per second, which by trial and error, was determined to be an adequate rate for allowing sediment to flush through the camera field of view, reducing the likelihood of imaging the same particles in successive images. For a typical 16 to 18 meter vertical profile, the data acquisition process with the FlocARAZI took approximately 15 minutes. For example, one such profile over 16 meters took 14 minutes. During that time, the FlocARAZI captured 1162 images. From those images 111,902 in focus particles where identified with 51,672 of those particles being larger than $30 \mu \mathrm{m}$. Taking data at this rate also requires a large amount of data storage space. For example, during a 9 day long survey, around 59,000 images were collected with the FlocARAZI, requiring nearly $700 \mathrm{~GB}$ of drive storage space. A typical image collected during the survey contained between 50 to 150 in focus particles. Observations with the FlocARAZI revealed $d_{50}$ floc sizes in the range of $70-130 \mu \mathrm{m}$ within the freshwater reaches of the Mississippi River Delta, with the largest flocs observed to be in the range of $400-500 \mu \mathrm{m}$ (Osborn et al., 2020).

\section{Estimating suspended sediment mass concentration from images}

The mass concentration of suspended sediment both in the field and laboratory often needs to be measured. In this section we present a method for extracting mass concentration measurements from images collected with the FlocARAZI that can be used to supplement physical water column samples. Because flocs can be distinguished from sand in these images, the method provides a means of estimating the mass of sand and mud separately under certain assumptions, something that cannot be accomplished with traditional laser or optical methods. The method cannot replace physical sampling because it needs to be calibrated. However, the method does provide a means of supplementing physical sampling. Here we first present the method that was developed in tandem with a controlled laboratory experiment. We then show the method applied to images and physical samples of SSC from the Mississippi River.

\subsection{Method overview}

To measure SSC one must know both the dry mass of the sediment within a suspension sample (or the dry volume and sediment density) and the total volume of the 
sample. To obtain SSC measures from images we estimate the volume of sediment imaged per volume of suspension sampled and then convert the sediment volume to a mass using a specified sediment density. For example, the average mass concentration, $C$, from a set of $n$ number of images can be defined as,

$$
C=\frac{\sum_{i=1}^{n} \sum_{j=1}^{k} \rho_{a, i j} \forall_{f, i j}}{n \forall_{I M}}
$$

where $k$ is the number of particles in each image, $\forall_{f, i j}$ is the $j$-th imaged floc or particle within the $i$-th image, $\rho_{a, i j}$ is the apparent density of that particular floc or particle, and $\forall_{I M}$ is the volume of the region imaged by the camera. Therefore, to estimate $C$, one must know the volume of the individual particles, the density of the particles, and volume of fluid being imaged.

The most straightforward of these three parameters to estimate is the particle or floc volume, $\forall_{f}$ :

$$
\forall_{f}=\frac{\pi}{6} d_{f}^{3}
$$

These volume estimates are made for each identified particle in each image using the projected area of the particle or flocs (Eq. 1). While equation 3 does not provide a true measure of particle volume due to the $2 \mathrm{D}$ nature of the images, it is reasonable to expect it to provide a non-biased estimate since particles are measured in suspension without any directional preference over thousands of particles.

Density estimates for each particle are more complicated to obtain. In each image, it is possible that identified particles could be a solid mineral (sand or silt particle), a porous to semi-porous mud aggregate or floc, or even organic and biological material such as fine particulate organic matter (FPOM) or plankton. The method presented here for estimating particle density does not account for organic or biological material. Though, if organic and biological material represent a small fraction of imaged particles, their effect on the overall SSC would be expected to be minimal. If solid mineral particles can be identified, then the density of those particles can be set to the density of silica sand ( $\rho_{a}=2650 \mathrm{~kg} / \mathrm{m}^{3}$ irrespective of particle size). However, estimating the density of the mud fraction is complicated by the irregular structure of mud flocs. The irregular and porous structure of flocs means that floc density can vary from floc to floc and for any individual floc with size. For example, the overall floc density has been shown to be a non-unique power function of floc size (Dyer \& Manning, 1999). Due to the irregular and compounding packing structure of flocs, and the measured power-law behavior of floc density with floc size, floc density is often modeled assuming the flocs are 3D fractal aggregates (Li \& Ganczarczyk, 1989; Flesch et al., 1999; Maggi et al., 2007) even though it is unlikely that flocs are strictly fractal in nature. Within this framework, the apparent floc density, $\rho_{a}$ (defined as the floc's dry mass divided by its wet volume), depends on the characteristic floc size and the density, size, and arrangement of the constituent primary particles that make up the floc:

$$
\rho_{a}=\rho_{0}\left(\frac{d_{f}}{d_{p}}\right)^{N_{f 3}-3}
$$

In equation $4, \rho_{0}$ is the density of the primary particles that make up the floc, $d_{f}$ is the equivalent spherical diameter of the floc, $d_{p}$ is the equivalent spherical diameter of the primary particles (Bowers et al., 2017), and $N_{f 3}$ is the 3D fractal dimension of the floc. The 3D fractal dimension is not a quantity that can be measured directly from two-dimensional (2D) images. Therefore, to obtain $N_{f 3}$, and hence floc density, we used the model developed by Maggi and Winterwerp (2004) for converting from a 2D perimeter-based fractal dimension to a 3D fractal dimension (see the appendix for details). This model calculates the 3D fractal dimension, $N_{f 3}$, using each measured floc perimeter and area. 
The last measure needed for the estimate of $C$ from equation 6 is the imaged volume, $\forall_{I M}$. A simple estimate of $\forall_{I M}$ can be obtained by multiplying the field of view by the sampling gap size or width created by the acrylic end caps of the camera and light housing (Fig. 2). However, the gap size between the acrylic end caps is larger than the camera's depth of field. This means that the volume associated with in-focus particles would be equal to or less than the physical gap size. Furthermore, the perceived imaged volume could be a function of imaged particle size. For example, larger particles could be more likely to be in focus within the depth of field of the camera compared to smaller particles that have a narrower margin to be in focus. To account for this phenomena, we developed a variable width for the imaged volume that is dependent on particle size. The variable width accounts for the smaller particles that are assumed to be within the imaged volume, but not in focus within the image. For our purposes, we took the variable sampling width, $w$, to be described by a logistic function where the width increases with particle size within a defined range, then asymptotically reaches a constant width:

$$
w\left(d_{f}\right)=\frac{W}{1+e^{-k\left(d_{f}-d_{0}\right)}}
$$

The constant width, $W$, should be taken to be the smaller of either the width of the gap or the depth of field of the camera, as either of these widths could limit the volume observable by the camera. We take the logistic growth rate, $k$, and the sigmoid's midpoint, $d_{0}$, as being experimentally determined values that are dependent on the depth of field of the particular camera and optics setup.

The average mass concentration, $C$, including the modification to the image volume by equation 5 is computed as:

$$
C=\sum_{i=1}^{n} \sum_{j=1}^{k} \frac{\rho_{a, i j} \forall_{f, i j}}{n L_{I M} H_{I M} w_{i j}}
$$

where $L_{I M}$ and $H_{I M}$ is the image width and height, respectively.

\subsection{Experiments to test and calibrate the method}

We ran a series of laboratory mixing tank experiments over a range of known SSC values and turbulent mixing rates to calibrate and test the methodology outlined above for estimating $C$ from images.

The experiments were conducted in the same aforementioned 13-liter mixing tank (e.g., Tran et al., 2018) (Fig. 3). For each run, a known mass of sonicated kaolinite clay was added to the mixing tank to obtain a known SSC. The concentrations tested include 20, 100, 200, 300, 400 and $500 \mathrm{mg} / \mathrm{L}$. An additional $5 \mathrm{~mL}$ of a $300 \mathrm{mg} / \mathrm{L}$ xanthan gum solution was added to the mixing tank to encourage flocculation. The experiments were conducted at two different turbulent shear rates, $G$, of approximately 50 and $90 \mathrm{~Hz}$. Before images were collected for the experiment, the flocs formed from the sonicated sediment were allowed to mix for three hours to ensure that an equilibrium size distribution had been reached. After three hours, the camera was set to record one image per second for 15 minutes. The same procedure was then repeated for the remaining mass concentrations and turbulent shear mixing rates. An optical backscatter sensor (OBS) was also placed within the tank to check for settling. Output from the OBS corroborated the conclusion suggested by visual inspection of the tank bottom; that being that no settling occurred in any of the test cases.

Images from the experiments were processed to provide floc size information for each floc identified within the set of collected images. This size information was then used in conjunction with the model developed by Maggi and Winterwerp (2004) to estimate a 3D fractal dimension for each floc. Assuming a characteristic primary particle density and size, the apparent density of each floc was estimated using equation 4 . The mass of 
each individual floc was then estimated by multiplying the apparent density by its equivalent spherical volume. For the analysis, the primary particle density was set as $2500 \mathrm{~kg} / \mathrm{m}^{3}$ and $d_{p}$ was taken to be $6.6 \mu \mathrm{m}$, which is the mean particle size of the kaolinite clay mixture as measured by the HORIBA particle size analyzer after sonication of the clay and sodium hexametaphosphate suspension. $\rho_{0}=2500 \mathrm{~kg} / \mathrm{m}^{3}$ was used instead of $\rho_{s}=$ 2600 or $2650 \mathrm{~kg} / \mathrm{m}^{3}$ in an attempt to account for the potential of aggregates of size 6.6 $\mu \mathrm{m}$ being slightly less dense than the raw mineral.

A summary of the experimental conditions and resulting floc size statistics at equilibrium is given in Table 2

Table 2. Average floc sizes for each concentration and mixing rate

\begin{tabular}{ccccc}
\hline $\begin{array}{c}\mathrm{SSC} \\
{[\mathrm{mg} / \mathrm{L}]}\end{array}$ & $\begin{array}{c}G \\
{[\mathrm{~Hz}]}\end{array}$ & $\begin{array}{c}d_{16} \\
{[\mu \mathrm{m}]}\end{array}$ & $\begin{array}{c}d_{50} \\
{[\mu \mathrm{m}]}\end{array}$ & $\begin{array}{c}d_{84} \\
{[\mu \mathrm{m}]}\end{array}$ \\
\hline 20 & 50 & 15 & 27 & 54 \\
20 & 90 & 15 & 34 & 62 \\
100 & 50 & 55 & 98 & 141 \\
100 & 90 & 30 & 43 & 58 \\
200 & 50 & 79 & 109 & 150 \\
200 & 90 & 49 & 67 & 93 \\
300 & 50 & 100 & 141 & 198 \\
300 & 90 & 54 & 77 & 108 \\
400 & 50 & 116 & 171 & 245 \\
400 & 90 & 64 & 94 & 132 \\
500 & 50 & 125 & 195 & 280 \\
500 & 90 & 68 & 104 & 151 \\
\hline
\end{tabular}

\subsection{Concentration estimation results}

For all test scenarios, SSC was estimated for each minute of the collected image series, both with and without the inclusion of the variable sampling volume width (Eq. 5). The SSC estimates without the inclusion of the variable width was used to inform the parameters of the variable width logistics equation, namely $k$ and the sigmoid's midpoint, $d_{0}$. The average of the estimated uncorrected SSC for each scenario is plotted in Figure 5a using a constant gap or sample volume width, $w$, against the known concentration. In all cases except the lowest two concentrations (20 and $100 \mathrm{mg} / \mathrm{L})$, the SSC estimates from the images are greater than the known SSC by a factor of $\approx 4.4$. That is, the relationship between $C$ estimated from the images and $C$ known through the addition of a known mass to the mixing tank appears to be linear across the majority of the concentrations.

The variable sampling width, $w\left(d_{f}\right)$ (Eq. 5), parameters of $k$ and $d_{0}$ were tuned to bring the lower concentration experiments more in line with the linear trend of the higher concentration experiments. The resulting sampling volume width equation that achieves this end is:

$$
w\left(d_{f}\right)=\frac{1.55}{1+e^{-0.2\left(d_{f}-27\right)}}
$$

Equation 7 is plotted in Figure 5b. The result of defining the sampling volume width using Equation 7 on the relationship between estimated SSC from the images and the known SSC is shown in Figure 5c. The line of best fit, with an intercept passing through the origin, has a slope of 4.5047 and a $R^{2}=0.9755$. It is this fit equation that is used to map originally estimated SSC to corrected SSC. Values of actual, original estimates, and corrected SSC are given in Table 3 along with the percent difference between the 

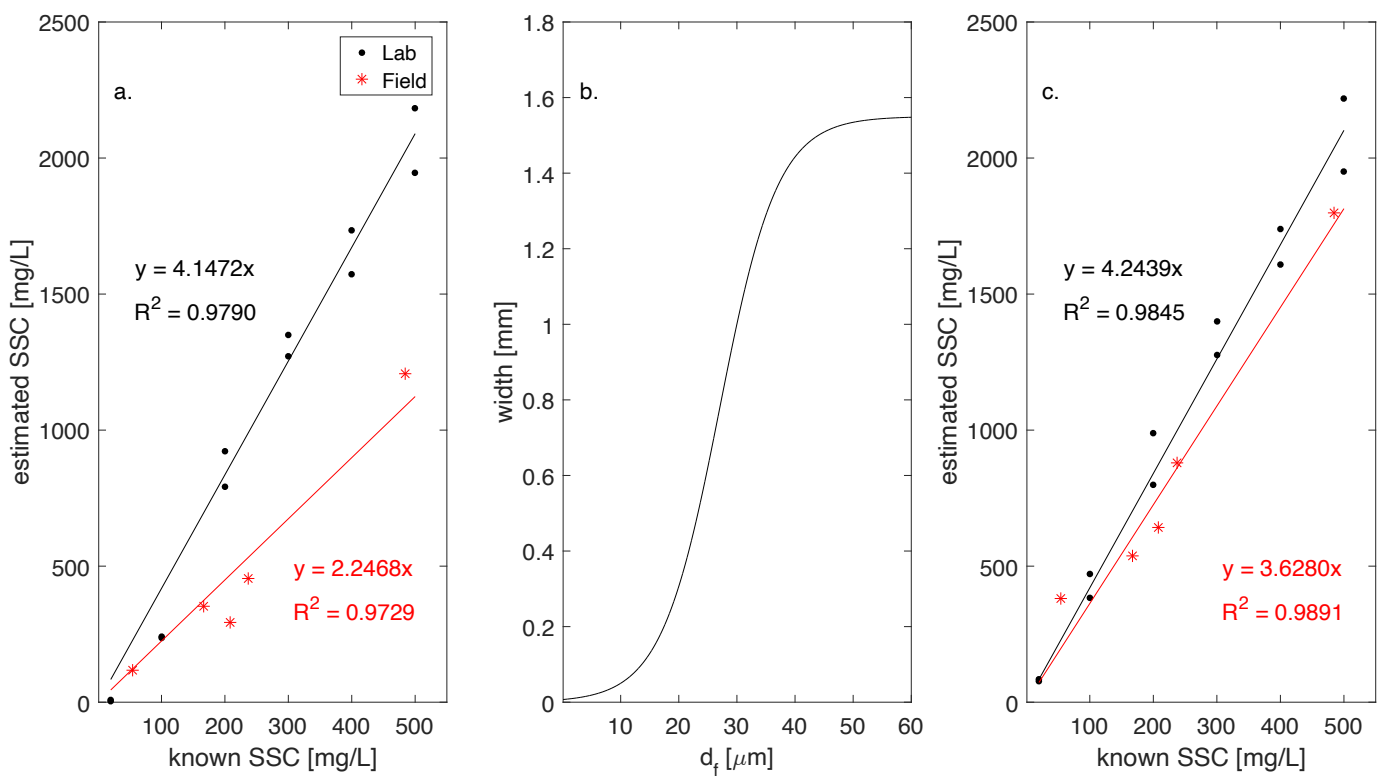

Figure 5. (a) SSC estimated using the camera particle data vs. known concentration in the mixing tank and field. (b) A variable width curve, dependent on floc size, used to account for particles outside of the cameras depth of field. (c) SSC estimated with the inclusion of the variable width curve. The slope of the best fit line, shown here, will be used as an additional correction factor to map the estimated SSC to a corrected SSC.

actual and image-based corrected SSC estimates. The standard deviations for the corrected SSC estimates only deviate slightly from the corrected SSC, revealing that concentration estimates for each minute only deviate slightly from the full 15 minute average.

\subsection{A field application}

We also performed a calibration of the image derived SSC using field data from the Mississippi River where we had paired image and physical concentration measurements over a vertical profile within the upper most portion of Southwest Pass from a January 2021 survey. The physical concentration measurements were collected with a US P-6 point sampler over a depth of approximately $20 \mathrm{~m}$. Approximately $1 \mathrm{~L}$ samples were collected at $5 \%, 25 \%, 50 \%, 75 \%$, and $95 \%$ of the flow depth with the P-6. The water samples were filtered on site with $1 \mu \mathrm{m}$ glass fiber filters and the liquid volume of the sample recorded. Once back to the lab, filtered water samples were allowed to dry in an oven at 80 degrees Celsius for 24 hours. The sample and filter were then weighed and the mass of the filter subtracted to obtain the mass of the sample. Mud concentrations over the depth at the location ranged from near $100 \mathrm{mg} / \mathrm{L}$ near the free surface down to almost $500 \mathrm{mg} / \mathrm{L}$ near the bed. Following the same process as outlined in the section above, we calibrated image-derived SSC both with and without a variable sampling width. The resulting calibration for each is shown in Figure 5 in red.

\subsection{SSC estimate dependence on $d_{p}$}

The two user-selected input parameters for the routine used to estimate SSC are the primary particle density, $\rho_{0}$, and the primary particle characteristic diameter, $d_{p}$. The models dependence on $\rho_{0}$ is linear. Consequently, changing the specified $\rho_{0}$ will linearly 
Table 3. SSC estimated from image data and linearly scaled corrected SSC estimate

\begin{tabular}{cccccc}
\hline $\begin{array}{c}\text { Actual } \\
\text { SSC } \\
{[\mathrm{mg} / \mathrm{L}]}\end{array}$ & $\begin{array}{c}\text { Mixing } \\
\text { rate } \\
{[\mathrm{Hz}]}\end{array}$ & $\begin{array}{c}\text { Estimated } \\
\text { SSC } \\
{[\mathrm{mg} / \mathrm{L}]}\end{array}$ & $\begin{array}{c}\text { Corrected } \\
\text { SSC } \\
{[\mathrm{mg} / \mathrm{L}]}\end{array}$ & $\begin{array}{c}\text { Corrected } \\
\text { SSC Std. } \\
{[\mathrm{mg} / \mathrm{L}]}\end{array}$ & \% diff. \\
\hline 20 & 50 & 83.4 & 18.5 & 0.8 & 7.4 \\
20 & 90 & 91.6 & 20.3 & 0.6 & 1.7 \\
100 & 50 & 450 & 99.8 & 2.1 & 0.2 \\
100 & 90 & 530 & 118 & 2.0 & 17.6 \\
200 & 50 & 831 & 184 & 3.8 & 7.8 \\
200 & 90 & 987 & 219 & 2.2 & 9.6 \\
300 & 50 & 1313 & 291 & 8.1 & 2.9 \\
300 & 90 & 1562 & 347 & 1.9 & 15.6 \\
400 & 50 & 1783 & 396 & 7.7 & 1.0 \\
400 & 90 & 1744 & 387 & 4.1 & 3.2 \\
500 & 50 & 2007 & 446 & 7.7 & 10.9 \\
500 & 90 & 2433 & 540 & 3.4 & 8.0 \\
\hline
\end{tabular}

scale the estimated SSC. However, the estimated SSC is not linearly dependent on $d_{p}$ (Eq. 4). The dependence of the uncorrected estimated SSC on $d_{p}$ was investigated for all test scenarios by observing the shape and magnitude of estimated SSC after setting $d_{p}$ equal to 2.6 and $10.6 \mu \mathrm{m}$ (Fig. 6). The general behavior of the model to a change in $d_{p}$ is a decrease in the magnitude of estimated SSC with a decrease in $d_{p}$, and an increase with increasing $d_{p}$. The linearity of the estimated SSC appears to hold for both choices of $d_{p}$. The slope of the line of best fit with an intercept passing through the origin is 2.58 $\left(R^{2}=0.9464\right)$ and $6.0422\left(R^{2}=0.9824\right)$ for $d_{p}=2.6$ and 10.6, respectively.

In the same way that the estimated SSC for the base scenario was scaled by the slope of the line of best fit through the SSC estimates, the SSC estimates produced with the changed $d_{p}$ were scaled by the slope of the line of best fit through the SSC estimates. The percent difference between the corrected SSC and the known SSC is presented in table 4 along with the base scenario with a $d_{p}$ of $6.6 \mu \mathrm{m}$. Reducing the primary particle size by $61 \%$ from $6.6 \mu \mathrm{m}$ to $2.6 \mu \mathrm{m}$ causes an average percent difference in corrected SSC of $11.4 \%$. The largest observed percent difference is $28.4 \%$, which occurred for a $d_{p}$ of $2.6 \mu \mathrm{m}$ for the $100 \mathrm{mg} / \mathrm{L}$ test at a mixing rate of $90 \mathrm{~Hz}$. Increasing $d_{p}$ by $61 \%$ to 10.6 $\mu \mathrm{m}$ results in an average percent difference in uncorrected SSC of $7.8 \%$. The average percent difference for the base case where $d_{p}=6.6 \mu \mathrm{m}$ is $7.2 \%$.

The model presented here for estimating SSC from particle data collected with the FlocARAZI camera system was developed by taking the characteristic primary particle diameter as the median particle size of the test sediment and assuming a density of $2500 \mathrm{~kg} / \mathrm{m}^{3}$. Since the proposed model includes linearly scaling the uncorrected SSC by a known SSC measurement, the input parameter $\rho_{0}$ has no consequence on the final corrected SSC output from the routine. Specifying $\rho_{0}$ simply provides a starting point for estimated SSC that will be corrected by a physical measurement, or to provide relative concentration estimates between a series of deployments with the FlocARAZI. The choice of $d_{p}$, however, influences both the magnitude and, to a lesser extent, the shape of estimated SSC as shown in figure 6. The difference in magnitude will be accounted for when linearly scaling the uncorrected SSC. Though, the difference in shape is not accounted for with the linear scaling. Therefore, the importance of the specified value for $d_{p}$ should be considered when a wide range of SSC and floc sizes are observed. The tests presented here show that within a range of $d_{p}$ values that could be reasonably observed in nature, the response of the model to changes in $d_{p}$ is close to linear. 
Table 4. Percent difference of linearly scaled corrected SSC estimates from the actual SSC for different values of $d_{p}$

\begin{tabular}{ccccc}
\hline Actual & Mixing & \multicolumn{3}{c}{$\%$ difference } \\
\cline { 3 - 5 } SSC & rate & \multicolumn{3}{c}{$d_{p}[\mu \mathrm{m}]$} \\
{$[\mathrm{mg} / \mathrm{L}]$} & {$[\mathrm{Hz}]$} & 2.6 & 6.6 & 10.6 \\
\hline 20 & 50 & 5.0 & 7.4 & 13.2 \\
20 & 90 & 17.4 & 1.7 & 5.7 \\
100 & 50 & 13.9 & 0.2 & 7.0 \\
100 & 90 & 28.4 & 17.6 & 12.4 \\
200 & 50 & 0.4 & 7.8 & 11.7 \\
200 & 90 & 17.4 & 9.6 & 5.7 \\
300 & 50 & 1.2 & 2.9 & 4.0 \\
300 & 90 & 22.8 & 15.6 & 12.0 \\
400 & 50 & 3.9 & 1.0 & 0.2 \\
400 & 90 & 0.9 & 3.2 & 5.2 \\
500 & 50 & 17.7 & 10.9 & 7.4 \\
500 & 90 & 7.4 & 8.0 & 8.5 \\
\hline
\end{tabular}

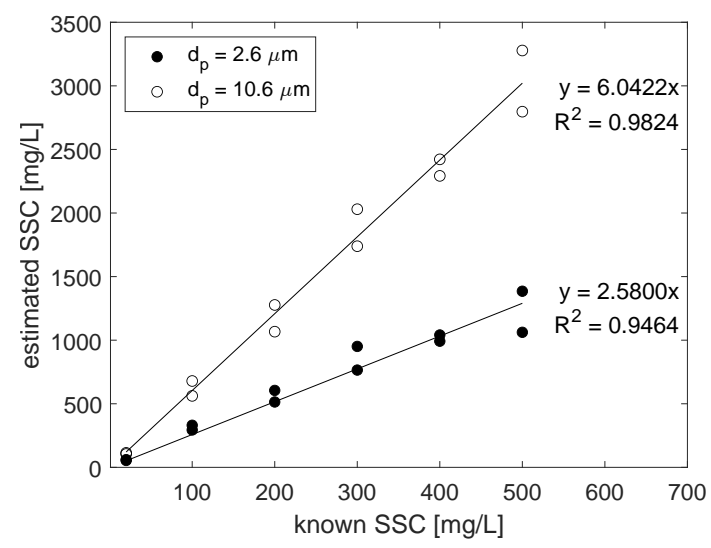

Figure 6. Estimated SSC and line of best fit for two different values of $d_{p}$ while maintaining $\rho_{0}=2500 \mathrm{~kg} / \mathrm{m}^{3}$. 


\section{Identifying sand within particle data}

When deploying the FlocARAZI in a high energy sand-bed river such as the Mississippi River, sand grains are likely to be in suspension and imaged as part of the suspended load along with flocs. Therefore, a method for identifying sand grains within the image data is desired to allow for analysis of the mud data separated from the complete data set.

To achieve the separation of sand particles from mud aggregates of similar size, we used an optimizable support vector machine (SVM) binary classifier within the MATLAB Classification Learner Toolbox. SVM is a supervised machine learning algorithm that attempts to classify data by finding the hyperplane that best separates the two classes. The hyperplane is a flat (n-1)-dimensional subspace within the n-dimensional space, where $\mathrm{n}$ is equal to the number of characteristics provided to the classifier to describe the data (Noble, 2006). For example, the characteristics from the particle data could include area, perimeter, aspect ratio, etc. In addition, SVM can separate nonlinear data by including a kernel function, where the kernel function allows for transforming the data to a higherdimension feature space and fitting a hyperplane within the higher-dimensional feature space. The resulting higher-dimensional hyperplane can then be mapped to the original n-dimensional space, resulting in a non-linear curve or non-flat surface separating data classes within the $n$-dimensional space, for $n=2$ and $n=3$ and greater, respectively. A trained SVM model can then classify new data based on which side of the curve or surface the new data is located.

\subsection{Data preparation}

Particle data from images collected both in the field and in the lab were used for the training and testing of the SVM. The field data is from images collected on the lower Mississippi River near the Bonnet Carré Spillway (BCS) in December 2019. This area of river is characterized by a subaqueous sand bar that runs adjacent to the BCS, which is positioned immediately downstream on the inside of a bedrock bend (Nittrouer et al., 2012). Images for this analysis were collected both over the thalweg within the bend and over the subaqueous sand bar. Laboratory data was included to train the model with an extended range of floc and sand data past what was observed in the field. The lab floc data came from the tests performed for the concentration estimation experiments. Additional sand particle data was obtained by sieving bed sediment, originating from the New River near Radford, VA, through seven mesh sieves, ranging in size from $63 \mu \mathrm{m}$ to $500 \mu \mathrm{m}$. Sand from each sieve was imaged and the processed data was included with the training data. Each particle from the lab and field images was then identified by eye as being either sand or not sand, and the row of data from the image processing routine associated with the identified particle was tagged with a one or zero, respectively. Any particle smaller than $63 \mu \mathrm{m}$ was considered to not be sand. The resulting combined data set consisted of 852 sand particles and 13,372 non-sand particles. The process of identifying the sand grains in this set of images took roughly 7 hours. The combined data was then split into training $(80 \%)$ and testing $(20 \%)$ data sets.

\subsection{Training and testing SVM classification model}

Training the optimizable SVM model consisted of importing the training data set into the MATLAB Classification Learner App, selecting a model validation method, selecting which features to train the model with, and optionally performing a principal component analysis on the selected features to reduce the feature space. In addition, optimizable components of the model include the kernel function, box constraint level, kernel scale, and standardization of the data. For the developed model, an initial Principal Component Analysis (PCA) revealed that the majority of information is provided by the area, perimeter, major and minor axis of an ellipse fit around the particles, and 
the contrast of the particle. Therefore, these features were selected as the training features, with the remaining features excluded. These five features were then passed through the PCA within the classification learner app, and three of the principal components were retained. The reduced particle data, within the principal component space is then used as the particle characteristic data that defines the location of sand and non-sand particles within the hyperspace. A Gaussian kernel function and the option to standardize the data were selected. The SVM box constraint level and kernel scale were selected as optimized parameters. That is, the Classification Learner App automatically selects and tests different combinations of values for the box constraint and kernel scale, and updates the optimized parameters based on a selected optimizer, in this case Bayesian optimization was selected. Finally, 5-fold cross-validation was implemented to gauge the accuracy of the model.

Table 5. Results from testing the trained SVM model on unseen particle data

\begin{tabular}{ccccc}
\hline $\begin{array}{c}\text { Particle } \\
\text { type }\end{array}$ & $\begin{array}{c}\text { \# of } \\
\text { particles }\end{array}$ & $\begin{array}{c}\text { \# correctly } \\
\text { identified }\end{array}$ & $\begin{array}{c}\text { True positive } \\
{[\%]}\end{array}$ & $\begin{array}{c}\text { False discovery } \\
{[\%]}\end{array}$ \\
\hline Floc & 2692 & 2689 & 99.89 & 0.15 \\
Sand & 153 & 149 & 97.39 & 1.97 \\
\hline
\end{tabular}

The resulting classification model had a $99.67 \%$ accuracy with the training set. Applying the model to the reserved test set produced an overall accuracy of $99.75 \%$ and a true positive rate of $97.39 \%$ and $99.89 \%$ for the identification of sand and non-sand, respectively (Table 5). The separated floc and sand particle size distributions from the test data set are presented in figure 7 .

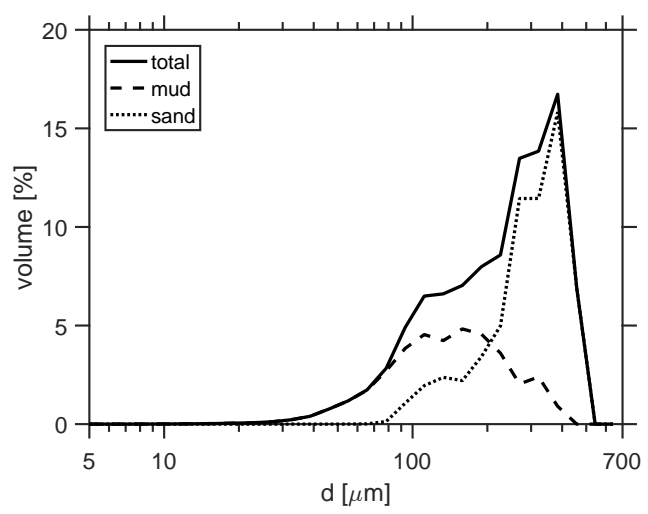

Figure 7. Volume-based particle size distributions for the floc and sand fraction and combined distribution. The distribution data is taken from the classification test data set.

\section{Discussion}

\subsection{FlocARAZI: range of conditions for deployment and comparison with other in-situ cameras}

In its current form, the FlocARAZI is suitable for imaging suspended particles in the size range of 5 to $600 \mu \mathrm{m}$. We have tested the system in both river and coastal ocean settings in depths from 0.25 to approximately $50 \mathrm{~m}$. The range of mean raw flow veloc- 
ities in these settings has ranged from roughly 0 to $2 \mathrm{~m} / \mathrm{s}$. However, what is more important than the raw velocity of the flow in determining image quality and data quantity is the relative difference in velocity between the camera system and the particles within the flow. Some movement of fluid is needed for a suspension to pass through the imaging window, but rapid movement of particles through the window also leads to particle streaking in the image (at least with the current camera, lens, and lighting). In the open ocean or lake, flow through the camera can be produced by the camera being lowered through the water column. In a river, the difference in velocity between the camera and suspension can be reduced by letting the camera and boat drift with the flow. In any vessel mounted environment, waves can present a significant challenge to high quality images as the wave action can cause the boat, and hence camera, to accelerate rapidly through pitch, roll, and heave. Adding weights to the camera frame helps to dampen, but not completely remove, the adverse effects of wave action.

We have used the camera in the lab and field in mud concentrations ranging from approximately 20 to $500 \mathrm{mg} / \mathrm{L}$. For these cases, a flow-through gap size, i.e., the distance between the acrylic end caps of the camera and light housing (Fig. 2), of $1.17 \mathrm{~mm}$ has worked well. In lower concentrations, such as the open ocean, it might be advantageous to increase the size of the flow-through gap to increase the number of particles captured in each image. Increasing the gap size might also be advantageous if particles on the order of a mm or larger in diameter are expected in suspension. For concentrations higher than $500 \mathrm{mg} / \mathrm{L}$, reducing the width of the gap might work to extend the range of concentrations in which images can be collected without significant overlapping of particles or flocs within the images that can cause bias in the size estimates from the image processing routines. A trade off with decreasing the gap size could be that more images are needed to produce a distribution in lower concentration environments and that the upper end of the measurable size range could start to be constrained by the physical gap width itself. For more discussion on bias caused through overlapping particles in the image and gap size see Tran et al. (2018).

The relatively simple nature of the construction of the FlocARAZI allowed for development of an in-situ particle sizing system that can be constructed with the cost of parts at less than $\$ 4000$ USD. The direct video stream to the surface provides the user the ability to know the condition of suspended sediment and image quality in real time. If images are out of focus, the stepper motor controlled platform, on which the camera is mounted, can be easily re-positioned while the FlocARAZI is deployed. With improvements in digital camera sensor technology over the past two decades, the FlocARAZI is able to collect high resolution $4000 \times 3000$ pixel images at a collection rate up to 10 frames per second with a compact and easy to handle form factor. Additionally, compared to the DFC, InSiPID, and PICS, which use a flashed light source that requires syncing with image acquisition, the FlocARAZI uses a continuously illuminated light source, reducing complexity and potential sources of equipment malfunction. Due to the compact nature of the system, a second camera and lens assembly could be added to the system with a different flow-through cell gap width, similar to the InSiPID camera system, to increase the range of observable particle sizes, depending on the intended application. However, as it stands a range of 5 to $600 \mu \mathrm{m}$ is a reasonably large range of particle or floc sizes when mud in high energy riverine and estuarine environments are of interest.

An additional benefit of the flow-through cell design utilized by the FlocARAZI is the ability to collect a large number of independent floc observations in a short period of time while profiling, when compared to imaging systems that rely on imaging flocs within a settling tube at discrete locations over the water column. For example, profiles with the PICS system have been reported to collect around 6,000 particle observations for a single profile consisting of seven sampling depths at a station with a total depth of 13.6 meters. This profile took approximately 22 minutes. In comparison, during a profile within the Mississippi River that took 14 minutes at a station with a depth of 16 me- 
ters, the FlocARAZI captured 1162 images and 111,902 in focus particles, with 51,672 of those particles being larger than $30 \mu \mathrm{m}$. While the FlocARAZI is capable of collecting a large number of independent floc observations in a short amount of time, the system is fundamentally limited in its ability to characterize flocs because it lacks the ability to measure the settling velocity of particles of a given size. This means that it cannot be used to obtain direct estimates of floc excess density along with floc size. Therefore, an ideal system for floc characterization might be to combine the FlocARAZI with a system like the PICS to obtain both a large number of independent floc size observations and floc settling velocity information.

\subsection{SSC obtained from images}

A common method for estimating SSC within aquatic environments is to relate SSC from physical samples to turbidity measured by an OBS. Others have estimated SSC with LISST instruments by simply assuming that the measured sediment only consists of dispersed minerals with a particular density. However, density becomes difficult to constrain in natural environments due to flocculation and the combining of different types and amounts of organic and mineral matter (Fall et al., 2021). In this paper, we have presented a method for estimating SSC from images. We see estimating concentration from the FlocARAZI images as a unique opportunity to have co-located size distributions, volume concentration estimates per size class, and total mass concentration associated with the mud and sand fractions.

To the best of our knowledge, only one other researcher has attempted to estimate SSC from a microscope-lens, image-based method (Antonenkov, 2016). Antonenkov (2016) developed an algorithm for identifying sand grains within images and related the pixel area of the sand grains to an equivalent circular diameter. The volume of the sand was then assumed to be equal to the volume of a sphere of the equivalent circular diameter and the mass was estimated by assuming a constant density for all sand grains. The SSC was then estimated as the sum of the mass of observed sand divided by the volume of the observable field of view for the collected images. This method corresponds exactly to the approach we take for estimating the contribution of sand to the total SSC estimate. Our method expands upon the work of Antonenkov (2016) to include a method for estimating SSC when flocs are present in suspension and for improving the accuracy of SSC estimates for particles in the smaller size ranges through the use of a variable sample width that is dependent on particle size.

While we see our image-based SSC estimation method as a step in the right direction, there are still significant limitations with the method that need to be considered. In particular, similar to estimates of SSC from a LISST, our image-based SSC estimation method suffers from a lack of a-priori information regarding the density of any given floc. We attempted to constrain the floc density problem through the use of fractal model for floc structure that relies on a single 3D fractal dimension and known properties of size and density of the primary particles of the flocs. While this may help to produce the overall general trends of decreasing floc density with size, the strict fractal model itself is still likely to be a flawed model for natural aggregates. Furthermore, even the density of the primary particles is difficult to measure, and it is not constrained to constant values or known relations (Fall et al., 2021). Added to these complications is the task of estimating a $3 \mathrm{D}$ fractal dimension from a $2 \mathrm{D}$ image, for which a well-tested method has yet to be developed.

The method we outline in the paper is our attempt to get as close as possible in our estimation of SSC from image data without directly using measured excess floc density from something like a settling test. Yet even if the models we employ, such as the fractal model for floc density, are correct, there is no way to obtain all of the needed parameters for these models from the image data alone. For this reason, the method can 
only be used as an estimate of SSC if it has been calibrated with physical water column samples or measures of floc settling velocity from the site of interest over the concentrations of interest. Use of a site-specific calibration equation (or the calibration factor since the relationship between image estimated SSC and measured SSC is a direct variation; see figure 5), accounts for a number of model parameters that have inherent and sitespecific variability. Such parameters include the empirically derived relation used to obtain $N_{f 3}$ from $N_{f 2}$, the use of a single characteristic $d_{p}$, estimating floc volume as the equivalent spherical volume, and variability in primary particle density.

Another limitation of the image-based SSC estimate is that it assumes that all of the suspended sediment is greater than $5 \mu \mathrm{m}$ in size (the lower limit of resolution associated with our current system). We've taken this to be reasonable for our suspensions given the visual high degree of flocculation in the lab and field, and given that the method over estimates concentration in general in all cases in which we've tested it. However, situations where flocculation is not significant and grain size is small (less than 5 to 10 $\mu \mathrm{m})$ could result in a significant fraction of the total suspended sediment mass not being captured in the estimate.

Further advances in estimating SSC from images will likely come from better models of floc density as a function of $2 \mathrm{D}$ shape and size. To obtain this, both measurement of density (possibly from settling velocity measurements) and measures of the three dimensional structure of flocs and how they related to their $2 \mathrm{D}$ projections are needed. The work of Fall et al. (2021) is a helpful example for considering how to combine data from multiple instruments to better constrained poorly measured components of the floc fractal model. Additionally, Pearson et al. (2021) recently developed a method to differentiate between components of the suspended mass associated with the sand and mud fraction by using a combination of sound and light scattering instruments. Comparison of data from such a system could help to better test and constrain the differentiated suspended mass from images making use of the sand and floc SVM segregation.

\subsection{Limitations and future use of the SVM for sand identification}

Observing suspended sediment characteristics with an image-based device provides the unique opportunity to visualize and characterize the particles that make up the calculated particle population statistics. Compared to non-optical particle sizing instruments, particle sizing with an image-based device such as the FlocARAZI provides the information necessary for training a machine learning algorithm for identifying sand within the population of observed particles. The ability to identify sand within the observed particle population is a significant advancement for floc research in fluvial environments where both flocs and sand are present in suspension. As a result, floc population characteristics can be calculated directly without making additional measurements and assumptions that would be necessary in the case of laser diffraction based particle sizing instruments or physical water samples where flocs are not observed directly.

We found great success with our particular SVM model over the conditions for which the model was trained and tested. Nevertheless, care should be taken when considering applying the model to other conditions. The identification of sand with the SVM model relies on the contrast of the identified particles and their geometric properties. Therefore, accurately distinguishing sand from other particles depends, to some extent, on the exposure conditions under which the images were taken. These can vary as a function of light and camera settings and the concentration and type of material in suspension. As a result, the SVM model should be checked for accuracy in each new deployment. Doing so would require choosing a subset of images and manually identifying mud flocs and sand, and then applying the SVM against the new data. If the results were unacceptable, the SVM model could be retrained with the addition of the newly obtained data included in the larger training dataset. With time, one would expect the SVM model 
to become more and more accurate and less dependent on the exact conditions of the camera and light at the time of deployment. However, at the moment we would not recommend the specific SVM model we have developed here be used in other locations or on other camera systems without adequate testing and updating as needed.

\section{Conclusions}

This paper presents the system components, packaging, and validation of an inexpensive and compact field-deployable camera system designed to image flocs in-situ. The use of an automated image processing routine and a method to obtain 3D fractal dimensions from 2D images were combined to estimate SSC of flocculated sediment from image data collected with the FlocARAZI. Additionally, a SVM machine learning model was introduced to identify sand grains within particle data collected with the FlocARAZI, providing the means to calculate floc size characteristics independent of suspended sand.

The FlocARAZI system and image processing code was designed to be a cost effective and easily reproducible solution for imaging flocs in-situ within the fluvial and marine environment. In accordance with the hope that developing a lower cost alternative to particle sizing instruments currently on the market will allow for an ever-increasing number of field observations of flocs. As part of this effort, the parts list for the FlocARAZI, build instructions, and image processing code are publicly available at https://doi.org/ 10.5281/zenodo.5541676 (the GitHub repository will be updated with content if the paper is accepted for publication).

Future work to improve the characterization of flocs and associated mass fluxes could come from combining data from the FlocARAZI (or similar image-based floc sizing instruments) with other measures of a flocs 3D structure and/or density. This could come through 3D imaging and massing of individual flocs and/or through collecting in situ settling velocity. Furthermore, making use of independent measures of the mud and sand concentration could help to improve the type and amount of data that can be collected with imaging systems.

\section{Appendix A Method for obtaining $N_{f 3}$ for each floc}

Maggi and Winterwerp (2004) developed their model for 3D fractal dimension based on $2 \mathrm{D}$ perimeter-based fractal dimension using data they generated by projecting $3 \mathrm{D}$ objects of specific 3D fractal dimension onto 2D coordinate planes. Included in their model is a resolution factor, $l$, that accounts for the fact that the imaged flocs are composed of individual pixels. A hyperbolic like equation was then fit to the data to produce a semiempirical relation for mapping $2 \mathrm{D}$ fractal dimensions to $3 \mathrm{D}$ fractal dimensions.

To use the method, the perimeter-based $2 \mathrm{D}$ fractal dimension, $N_{f 2}$, is computed as:

$$
N_{f 2}=2 \frac{\log [p]}{\log [A]}
$$

where the perimeter, $p$, and area, $A$, of the individual flocs are in pixels and pixels ${ }^{2}$ as obtained in the image processing output.

Next, the resolution factor, $l$, defined as the pixel length of one side of a square box surrounding an individual floc, is computed. However, since most flocs are bound by a rectangular box, $l$ is obtained by calculating the side length of a square of the equivalent area of the rectangular box surrounding the floc. That is,

$$
l=\sqrt{p_{x} p_{y}}
$$

where $p_{x}$ and $p_{y}$ are the length and width in pixels of the bounding rectangular box around an individual floc, both of which are outputs of the processing routine. In creating the 
mapping function, boundary conditions, $z(l)$ and $k(l)$, are introduced to account for the $N_{f 2}=(3, z(l))$ and $N_{f 3}=(k(l), 2)$ boundaries, respectively. The boundary condition, $z(l)$, is defined as the $2 \mathrm{D}$ fractal dimension of the projection of a box that has a resolution of $l$ :

$$
z(l)=\frac{\log [4 l-4]}{\log [l]}
$$

$k(l)$ is defined simply as a function of $z(l)$ by fitting data points at the boundary $N_{f 2}=$ 2 :

$$
k(l)=k(z(l))=z(l)[z(l)-1]+1
$$

A function of the form,

$$
N_{f 2}=\frac{a}{N_{f 3}^{2}}+b
$$

was then solved, resulting in the coefficients $a$,

$$
a(l)=9\left(z(l)-\frac{2[k(l)]^{2}-9 z(l)}{[k(l)]^{2}-9}\right)
$$

and $b$,

$$
b(l)=\frac{2[k(l)]^{2}-9 z(l)}{[k(l)]^{2}-9}
$$

where $N_{f 3}$ is the $3 \mathrm{D}$ fractal dimension. The $3 \mathrm{D}$ fractal dimension of an individual floc can then be estimated by:

$$
N_{f 3}=\sqrt{\frac{a(l)}{N_{f 2}-b(l)}} \text { for } \quad N_{f 2}<2
$$

Once $N_{f 3}$ is calculated for each floc, using equations A1-A8, the density of each floc, needed in equation 6 , can be calculated from equation 4 .

\section{Acknowledgments}

Funding for this work was provided by the National Science Foundation under EAR award 1801142, "Collaborative Research: Flocculation Dynamics in the Fluvial to Marine Transition." Additional financial support for R.O. was provided by the Charles E. Via, Jr. Endowment at Virginia Tech and the New Horizons Graduate Scholars Program. Three anonymous reviewers are greatly acknowledged for providing comments that help to improve the paper. Upon publication, data associated with this study will be available at https://doi.org/10.5281/zenodo.5541676 (the GitHub repository will be updated with content if the paper is accepted for publication), or by contacting the corresponding author (strom@vt.edu).

\section{References}

Agrawal, Y. C., \& Pottsmith, H. C. (2000). Instruments for particle size and settling velocity observations in sediment transport. Marine Geology, 168, 89-114.

Antonenkov, D. A. (2016). Method of the Aquatic Environment Image Processing for Determining the Mineral Suspension Parameters. Physical Oceanography, 5 .

Benson, T., \& French, J. R. (2007). InSiPID: A new low-cost instrument for in situ particle size measurements in estuarine and coastal waters. Journal of Sea Research, 58, 167-188.

Bowers, D. G., McKee, D., Jago, C. F., \& Nimmo-Smith, W. A. M. $\quad$ (2017). The area-to-mass ratio and fractal dimension of marine flocs. Estuarine, Coastal and Shelf Science, 189, 224-234. 
Cartwright, G. M., Friedrichs, C. T., \& Sanford, L. P. (2011). In situ characterization of estuarine suspended sediment in the presence of muddy flocs and pellets. In The Proceedings of the Coastal Sediments 2011 (pp. 642-655). World Scientific Publishing Company.

Chapalain, M., Verney, R., Fettweis, M., Jacquet, M., Le Berre, D., \& Le Hir, P. (2019). Investigating suspended particulate matter in coastal waters using the fractal theory. Ocean Dynamics, 69(1), 59-81.

Curran, K. J., Hill, P. S., \& Milligan, T. G. (2003). Time variation of floc properties in a settling column. Journal of Sea Research, 49(1), 1-9.

Davies, E. J., \& Nepstad, R. (2017). In situ characterisation of complex suspended particulates surrounding an active submarine tailings placement site in a Norwegian fjord. Regional Studies in Marine Science, 16, 198-207.

Droppo, I., \& Ongley, E. (1994). Flocculation of suspended sediment in rivers of southeastern Canada. Water Research, 28(8), 1799 - 1809.

Dyer, K. R., \& Manning, A. J. (1999). Observation of the size, settling velocity and effective density of flocs, and their fractal dimensions. Journal of Sea Research, $41,87-95$.

Eisma, D. (1986). Flocculation and de-flocculation of suspended matter in estuaries. Netherlands Journal of Sea Research, 20, 183 - 199.

Fall, K. A., Friedrichs, C. T., Massey, G. M., Bowers, D. G., \& Smith, S. J. (2021). The Importance of Organic Content to Fractal Floc Properties in Estuarine Surface Waters: Insights From Video, LISST, and Pump Sampling. Journal of Geophysical Research: Oceans, 126, 1-25.

Fennessy, M. J., Dyer, K. R., \& Huntley, D. A. (1994). inssev: An instrument to measure the size and settling velocity of flocs in situ. Marine Geology, 117, $107-117$.

Ferguson, R., \& Church, M. (2004). A simple universal equation for grain settling velocity. Journal of Sedimentary Research, 74 (6), 933-937.

Flesch, J. C., Spicer, P. T., \& Pratsinis, S. E. (1999). Laminar and turbulent shearinduced flocculation of fractal aggregates. AIChE Journal, 45, 1114-1124.

Graham, G. W., Davies, E. J., Nimmo-Smith, W. A. M., Bowers, D. G., \& Braithwaite, K. M. (2012). Interpreting lisst-100x measurements of particles with complex shape using digital in-line holography. Journal of Geophysical Research: Oceans, 117 (C5), C05034.

Hill, P. S., Boss, E., Newgard, J. P., Law, B. A., \& Milligan, T. G. (2011). Observations of the sensitivity of beam attenuation to particle size in a coastal bottom boundary layer. Journal of Geophysical Research: Oceans, 116, 1-14.

Karageorgis, A., Georgopoulos, D., Gardner, W., Mikkelsen, O., \& Velaoras, D. (2015). How schlieren affects beam transmissometers and LISST-Deep: an example from the stratified Danube River delta, NW Black Sea. Mediterranean Marine Science, 16, 366-372.

Keyvani, A., \& Strom, K. (2013). A fully-automated image processing technique to improve measurement of suspended particles and flocs by removing out-offocus objects. Computers and Geosciences, 52, 189-198.

Kumar, R. G., Strom, K. B., \& Keyvani, A. (2010). Floc properties and settling velocity of san jacinto estuary mud under variable shear and salinity conditions. Continental Shelf Research, 30, 2067 - 2081.

Li, D. H., \& Ganczarczyk, J. (1989). Fractal geometry of particle aggregates generated in water and wastewater treatment processes. Environ. Sci. Technol., 23, 1385-1389.

MacDonald, I. T., \& Mullarney, J. C. (2015). A Novel FlocDrifter Platform for Observing Flocculation and Turbulence Processes in a Lagrangian Frame of Reference. Journal of Atmospheric and Oceanic Technology, 32, 547-561.

Maggi, F., Mietta, F., \& Winterwerp, J. C. $\quad$ (2007). Effect of variable fractal dimension on the floc size distribution of suspended cohesive sediment. Journal 
of Hydrology, 343, 43-55.

Maggi, F., \& Winterwerp, J. C. (2004). Method for computing the three-dimensional capacity dimension from two-dimensional projections of fractal aggregates. Physical Review E, 69, 1-8.

Manning, A. J., \& Dyer, K. R. (2002). The use of optics for the in situ determination of flocculated mud characteristics. J. Opt. A: Pure Appl. Opt., 4, S71S81.

Markussen, T. N., Elberling, B., Winter, C., \& Andersen, T. J. (2016). Flocculated meltwater particles control arctic land-sea fluxes of labile iron. Scientific Reports, 6(1), 24033.

Mietta, F., Chassagne, C., \& Winterwerp, J. C. (2009). Shear-induced flocculation of a suspension of kaolinite as function of $\mathrm{pH}$ and salt concentration. Journal of Colloid and Interface Science, 336, 134-141.

Mikkelsen, O. A., Hill, P. S., \& Milligan, T. G. (2006). Single-grain, microfloc and macrofloc volume variations observed with a LISST-100 and a digital floc camera. Journal of Sea Research, 55, 87-102.

Mikkelsen, O. A., Hill, P. S., Milligan, T. G., \& Chant, R. J. (2005). In situ particle size distributions and volume concentrations from a LISST-100 laser particle sizer and a digital floc camera. Continental Shelf Research, 25, 1959-1978.

Mikkelsen, O. A., Milligan, T. G., Hill, P. S., Chant, R. J., Jago, C. F., Jones, S. E., ... MitchelsonJacob, G. (2008). The influence of schlieren on in situ optical measurements used for particle characterization. Limnology and Oceanography: Methods, 6, 133-143.

Mikkelsen, O. A., Milligan, T. G., Hill, P. S., \& Moffatt, D. (2004). INSSECT an instrumented platform for investigating floc properties close to the seabed. Limnology and Oceanography: Methods, 2, 226-236.

Nittrouer, J. A., Best, J. L., Brantley, C., Cash, R. W., Czapiga, M., Kumar, P., \& Parker, G. (2012). Mitigating land loss in coastal Louisiana by controlled diversion of Mississippi River sand. Nature Geoscience, 5, 534-537.

Noble, W. S. (2006). What is a support vector machine? Nature Biotechnology, 24, $1565-1567$.

Osborn, R., Dunne, K. B. J., Abolfazli, E., Strom, K., \& Nittrouer, J. A. (2020, December). Characterization of In-Situ Floc Sizes Over the Vertical Within the Mississippi River and its Distributaries. In Abstract [ep001-0005] presented at 2020 fall meeting. AGU.

Pearson, S. G., Verney, R., van Prooijen, B. C., Tran, D., Hendriks, E. C. M., Jacquet, M., \& Wang, Z. B. (2021). Characterizing the composition of sand and mud suspensions in coastal and estuarine environments using combined optical and acoustic measurements. Journal of Geophysical Research: Oceans, $126(7)$, e2021JC017354.

Schneider, C. A., Rasband, W. S., \& Eliceiri, K. W. (2012). Nih image to imagej: 25 years of image analysis. Nature Methods, 9, 671-675.

Smith, S. J., \& Friedrichs, C. T. (2011). Size and settling velocities of cohesive flocs and suspended sediment aggregates in a trailing suction hopper dredge plume. Continental Shelf Research, 31, S50-S63.

Smith, S. J., \& Friedrichs, C. T. (2015). Image processing methods for in situ estimation of cohesive sediment floc size, settling velocity, and density. Limnology and Oceanography: Methods, 13, 250-264.

Tran, D., Kuprenas, R., \& Strom, K. (2018). How do changes in suspended sediment concentration alone influence the size of mud flocs under steady turbulent shearing? Continental Shelf Research, 158, 1-14.

Tran, D., \& Strom, K. (2017). Suspended clays and silts: Are they independent or dependent fractions when it comes to settling in a turbulent suspension? Continental Shelf Research, 138, 81-94.

Zack, G. W., Rogers, W. E., \& Latt, S. A. (1977). Automatic measurement of sister 
chromatid exchange frequency. Journal of Histochemistry and Cytochemistry, 25, 741-753.

Zhu, Z., Yu, J., Wang, H., Dou, J., \& Wang, C. (2015). Fractal Dimension of Cohesive Sediment Flocs at Steady State under Seven Shear Flow Conditions. Water, 7, 4385-4408. 